\title{
Modelling pressure distribution in sonicated ethanol solution using COMSOL simulation
}

\author{
Nur Amira Hasnul Hadi ${ }^{1, *}$, Arshad Ahmad ${ }^{1}$, and Olagoke Oladokun ${ }^{1}$ \\ ${ }^{1}$ Department of Chemical Engineering/Centre of Hydrogen Energy, Faculty of Chemical and Energy \\ Engineering, Universiti Teknologi Malaysia, 81310 Johor Bahru, Johor, Malaysia.
}

\begin{abstract}
Ultrasound application has been reported to assist chemical processes as a result of various physiochemical effects during acoustic cavitation phenomena in a liquid. In this study, acoustic pressure distribution in ethanol solution induced by ultrasonic waves in a sonoreactor was investigated using COMSOL Multiphysics software. The variations of acoustic pressure distribution in ethanol liquid were investigated through a single-phase incompressible model developed by varying the frequency of an ultrasonic transducer. The simulation in COMSOL Multiphysics shows that the acoustic wave emitted from the bottom of the sonoreactor generated multiple layers of high acoustic pressure distribution. The fluctuating pressure magnitude along the sonoreactor shows that constructive interference produced high acoustic pressure region whereas destructive interference resulted in low acoustic pressure. Meanwhile, the distance over sound wave can travel before attenuation occurs is much further at $60 \mathrm{kHz}$. These results support the theory that wave attenuation is strongly frequency dependent.
\end{abstract}

\section{Introduction}

The potential of ultrasound as an alternative source of energy in process intensification has witnessed a great development in the chemical engineering industry. Several applications using ultrasound reported in the literature including chemical synthesis, green chemistry, solvent extraction, and separation are well-established laboratory operations [1, 2]. Despite the wide-ranging research at a laboratory scale, there is a limited number of chemical processing applications that are carried out on the industrial scale, mainly due to the lack of expertise, especially in proper reactor design and scale-up strategies. When ultrasound acts upon a liquid, bubbles are generated from acoustic cavitation phenomena where liquids are pulled apart by the applied frequency. This mechanism also produces free radicals via sonolysis process to assist various chemical reactions [3]. Generally, the propagation of ultrasound in a liquid will affect pressure balance inside a sonochemical reactor. Local existence of cavitational events near surface irradiation leads to the dissipation of energy in a bulk liquid where problem occurs. One step towards solving the problem is to develop a pilot-scale reactor and understand the spatial distribution of pressure in the reactor using modelling and simulation [4]. The use of modelling and simulation allows cavitational activities to be quantified using pressure distribution and fluid flow in order to identify active and passive zones. Most of the previous studies related to pressure distribution in a sonochemical reactor are limited to water. However, the present work discusses the characteristics of sonicated ethanol solution, a substance that exists in many azeotropic mixtures. The objective of this study is to investigate the acoustic pressure

*Corresponding author: $\underline{\operatorname{arshad} @ \text { utm.my }}$ 
distribution in a rectangular sonoreactor using ethanol as the medium of propagation at 20 , 40, and $60 \mathrm{kHz}$ using COMSOL Multiphysics software.

\section{Methodology}

\subsection{COMSOL simulation}

An ultrasonic system consisted of a transducer and a sonoreactor filled with ethanol solution was simulated as shown in Figure 1. The calculation model was a rectangular reactor with the dimensions of $70 \mathrm{~mm} \times 70 \mathrm{~mm} \times 200 \mathrm{~mm}$ and the thickness of $3 \mathrm{~mm}$. A transducer with a radius $20 \mathrm{~mm}$ was attached to the bottom of the reactor with varying frequencies of 20,40 , and $60 \mathrm{kHz}$. The piezoelectric material in the transducer converted electric current to sound field and passed through the sonoreactor [5]. The emitted amplified ultrasonic wave propagated through ethanol in the reactor. Two different modules were selected to simulate this physical effect in COMSOL Multiphysics software (version 5.3a): (1) the piezoelectric module for a transducer and (2) the acoustic structure interaction module. Each of this module was governed by its own equation that defined the specific physics as discussed in the following section.

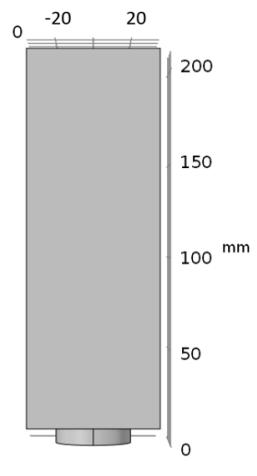

(a)

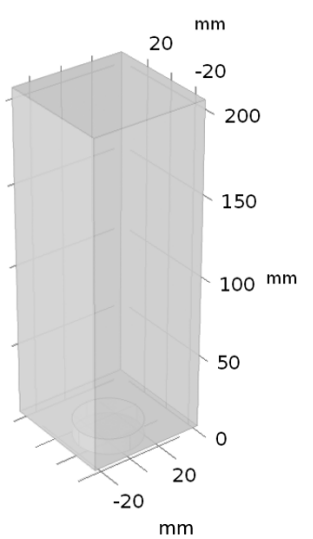

(b)

Fig. 1. Illustration of modelling an ultrasonic system: (a) side view and (b) three-dimensional view of a sonoreactor.

\subsection{Acoustic pressure field}

A wave equation describes wave propagation of an ultrasonic wave through a liquid medium, which is ethanol in this case. A second-order partial differential equation was used as the sound wave equation [5]. This equation expresses acoustic pressure $(p)$ as a function of $r$ and time $t$. The propagation of sound wave in one dimension ( $x$ direction) is defined by Equation 1:

$$
\frac{\partial^{2} p}{\partial x^{2}}-\frac{1}{c^{2}} \frac{\partial^{2} p}{\partial t^{2}}=0
$$


Where $p$ is the acoustic pressure $\left(\mathrm{N} \mathrm{m}^{2}\right)$ and $c$ is the speed of sound in medium $\left(\mathrm{m} \mathrm{s}^{-1}\right)$. Assuming the speed of sound is constant, the acoustic pressure is described as a function of ultrasound frequency as follows:

$$
p=p_{o} \sin (\omega t \pm k x)
$$

Where $\omega$ is the angular frequency and $k$ is the wave number. Using Equation 1, threedimensional wave equations can be written as:

$$
\begin{gathered}
\Delta^{2} \rho \frac{1}{\rho}-\frac{1}{c^{2} \rho} \frac{\delta^{2} p}{\delta t^{2}}=0 \\
\Delta^{2}=\frac{\partial^{2} p}{\partial x^{2}}+\frac{\partial^{2} p}{\partial y^{2}}+\frac{\partial^{2} p}{\partial z^{2}}
\end{gathered}
$$

Several assumptions were made in order to follow the equation above in the propagation of a liquid medium. The assumptions are: (a) linear propagation of sound wave through a medium, (b) shear stress is negligible (c), density and compressibility of liquid medium are constant, and (d) pressure is time harmonic. From all of the assumptions made, the Helmholtz equation is obtained as in Equation 5:

$$
\frac{\nabla^{2} p}{\rho}-\frac{\omega^{2}}{\rho c^{2}} p=0
$$

\subsection{Boundary conditions}

The following boundary conditions were applied during the simulation of the geometries: (a) the whole ultrasound energy entered the bottom of the sonoreactor through the above surface of the transducer where $p=p_{o}$ and $p_{o}$ is the initial amplitude of harmonic source, and (b) pressure amplitude vanished near the wall.

\section{Results and discussion}

Figure 2 shows acoustic pressure distribution along the reactor from the simulation results. The acoustic pressure magnitude was indicated by a colour scale. From the results, high-pressure field was observed just above the transducer's surface. During sonication, bubbles were formed via nucleation coming from existing bubbles in the fluid. The sound field caused the liquid to experience cycles of high (compression) and low (rarefaction) pressure phases, forcing these bubbles to expand and contract [6]. Since these phenomena induced acoustic bubbles, it was considered that acoustic pressure attenuated rapidly in a short distance from the transducer. 


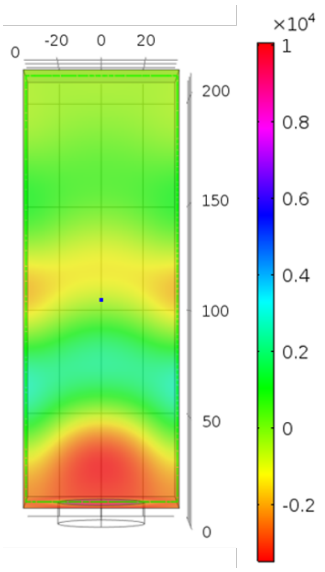

(a)

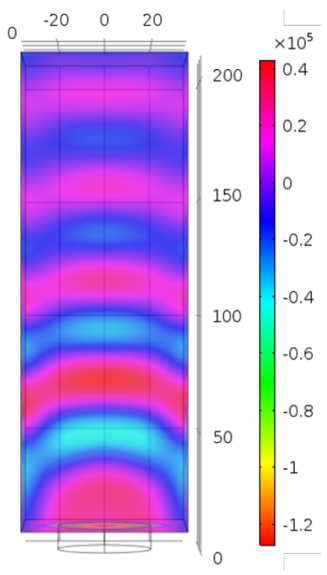

(b)

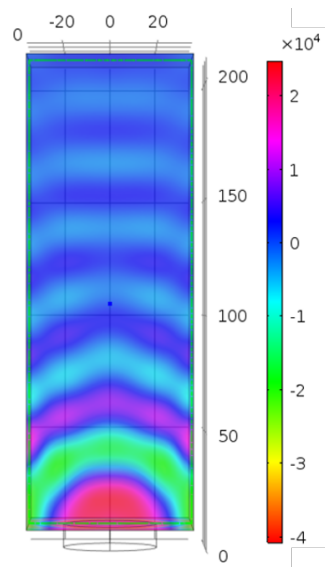

(c)

Fig. 2. Acoustic pressure distribution in the sonoreactor at (a) 20, (b) 40 (c), and $60 \mathrm{kHz}$.

As shown in Figure 2, the propagation of ultrasonic wave pattern became clearer when the frequency increased and ripples were formed. As the acoustic pressure decreased from the centre of the transducer to the liquid surface, the colour changed from red to blue. The simulation suggests that the effect of constructive/destructive interference is induced by wave interaction.

Figures 3 and 4 present the acoustic pressure distribution along the sonoreactor height at 40 and $60 \mathrm{kHz}$, respectively. The region closest to the surface of the transducer to a few $\mathrm{mm}$ height of the sonoreactor exhibited the highest increment of acoustic pressure compared to the upper region. The fluctuating pressure magnitude along the sonoreactor indicated constructive interference produced a high acoustic pressure region whereas destructive interference produced a low acoustic pressure region. As the wave continued to propagate throughout the reactor, the amplitude became smaller due to the sound scattering in the propagating medium [7]. The ultrasonic wave continued to travel until it reached the liquid surface where the wave was reflected along the incoming direction.

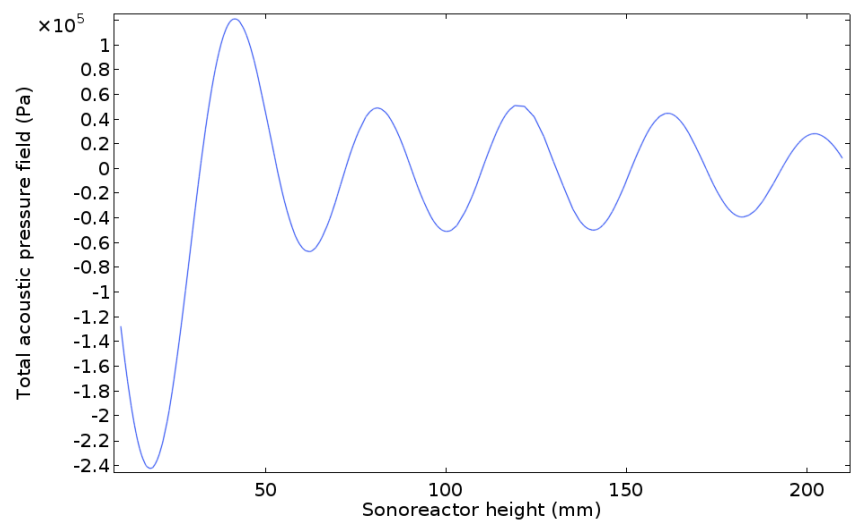

Fig. 3. Total acoustic pressure in ethanol along the sonoreactor height at $40 \mathrm{kHz}$. 


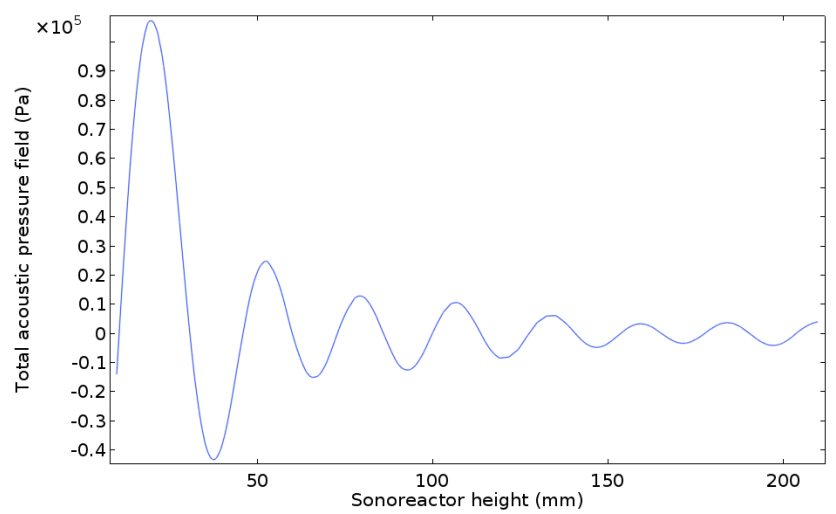

Fig. 4. Total acoustic pressure in ethanol along the sonoreactor height at $60 \mathrm{kHz}$.

At $40 \mathrm{kHz}$, the distance between pressure rarefaction and wavelength was slightly bigger compared to $60 \mathrm{kHz}$. Meanwhile, the distance over the sound wave could travel before attenuation occurred was much further at $60 \mathrm{kHz}$. From both figures, oscillation energy was less absorbed until it had reached the top wall of the sonoreactor before the waves started to dissipate. It can be concluded that attenuation is strongly frequency dependent. Vertical pressure distribution and horizontal propagation of acoustic pressure in ethanol are also depicted in Figure 5.

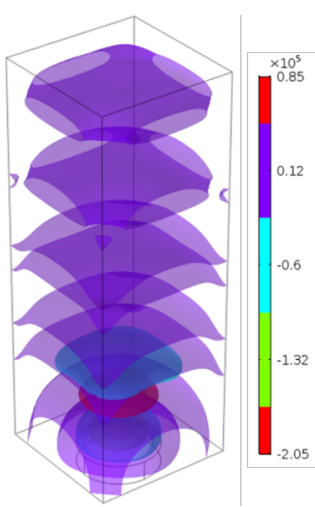

(a)

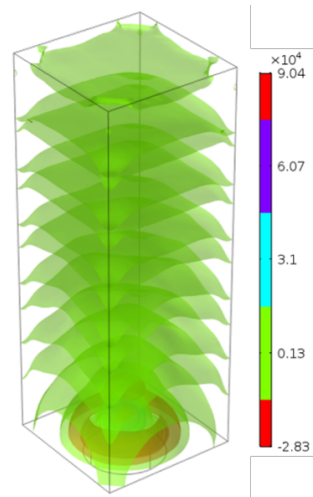

(b)

Fig. 5. Acoustic pressure waveform propagation across the sonoreactor at (a) 40 and (b) $60 \mathrm{kHz}$.

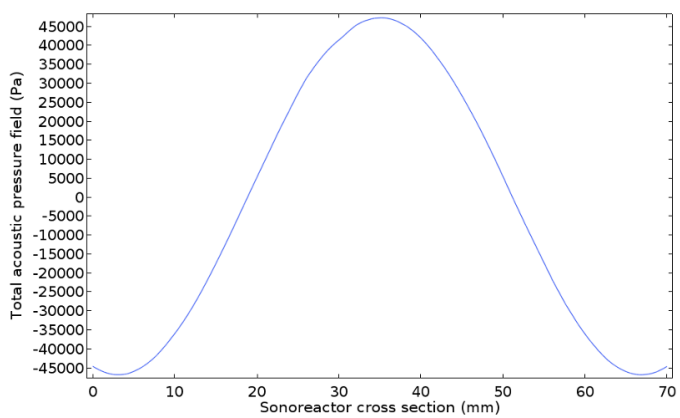

(a)

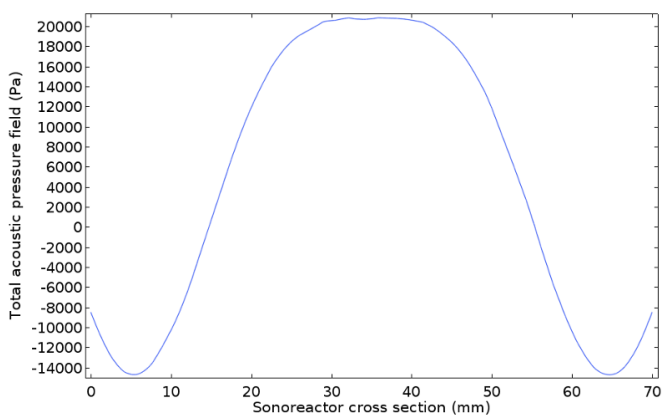

(b)

Fig. 6. Acoustic pressure propagation across the sonoreactor (a) 40 and (d) $60 \mathrm{kHz}$. 
Figures 5(a) and (b) indicate the waveform propagation of acoustic pressure during sonication at 40 and $60 \mathrm{kHz}$. Since $60 \mathrm{kHz}$ is considered as high frequency, oscillation is strongly absorbed and causing the wave to dissipate rapidly in liquid waveform. The propagation of acoustic pressure along ethanol solution stretched to the edges compared to $40 \mathrm{kHz}$ where waveform dissipated after travelling of almost a full distance in the sonoreactor. Meanwhile, at the centre of the sonoreactor, the wave propagation produced by the transducer in ethanol solution gave a quite different character of waveform, with a much wider peak (Figure 6(b)) at $60 \mathrm{kHz}$. The wider peak at $60 \mathrm{kHz}$ over $40 \mathrm{kHz}$ decayed more rapidly with distance, supporting the results in Figure 4 where there was an agglomeration of amplitude at higher frequency as the propagation distance increased [8].

\section{Conclusion}

The objective of this study was to investigate the acoustic pressure distribution in a rectangular sonoreactor using ethanol as the medium of propagation at 20,40 , and $60 \mathrm{kHz}$ using COMSOL Multiphysics software. The following conclusions can be drawn from the study. Firstly, this study has shown that the acoustic wave emitted from the bottom of the sonoreactor generates multiple layers of acoustic pressure with the highest pressure located at the bottom. The analysis of pressure distribution demonstrates that the existence of highand low-pressure acoustic fields is due to the wave interference across the reactor during sonication. At the highest frequency, the waveform of acoustic pressure clearly shows no sign of dissipation; however, the wave decays as the propagation distance increases. The objective of this study has been achieved. Although the overall pressure distribution is accordingly expected, an accurate result from experimental measurement must be made and compared with modelling. Kumar et al. suggested to couple the behaviour of acoustic cavitation bubble dynamic in a reactor with the wave equation in simulation [9]. Therefore, incorporating necessary properties such as cavitation bubbles and heat formation during cavitation are crucial to improve the simulation results.

This work was funded by Universiti Teknologi Malaysia and Malaysia-Thailand Joint Authority (MTJA) grant (Vot. No. 4C116).

\section{References}

[1] Richard James Wood, Judy Lee, Madeleine J. Bussemaker, Ultrasonic Sonochemistry, 38, 351-370 (2017)

[2] Taha Mahdi, Arshad Ahmad, Mohamad Mahmoud Nasef, Adnan Ripin, Separation \& Purification Reviews, 44:4, 308-330(2014)

[3] Franz Grieser, Muthupandian Ashokkumar, Advances in Colloid and Interface Science, 89-90, 423-438 (2001)

[4] Zongsu Wei, Linda K. Weavers, Ultrasonic Sonochemistry, 31, 490-498 (2016)

[5] Vinayak S. Sutkar, Parag R. Gogate, Levente Csoka, Chemical Engineering Journal, 158, 290-295, (2010)

[6] Muthupandian Ashokkumar, Ultrasonic Sonochemistry, 4, 864-872 (2011)

[7] Zheng Xu, Keiji Yasuda, Shinobu Koda, Ultrasonic Sonochemistry, 20, 452-459 (2013)

[8] Vinayak S. Sutkar, Chemical Engineering Journal, 158, 296-304 (2010)

[9] Peeush Kumar, Swati Khanna, Vijayanand S. Moholkar, Transport Phenomena and Fluid Mechanics, AIChe Journal, 58, 3858-3866 (2012) 